\title{
Parallel Operation of Hybrid Loaded Resonant Converter Using Phase-Shift Control
}

\author{
Taufik, Member, IEEE and James J. Mullins, Member, IEEE \\ California Polytechnic State University, San Luis Obispo \\ taufik@calpoly.edu
}

\begin{abstract}
This paper presents a phase shifting method to adjust the combined output voltage of two Hybrid Loaded Resonant Converters connected in parallel. The method implements a fixed switching frequency and hence it is particularly useful in applications such as medical instrumentation where audible noise is not desirable. The design and analysis of parallel Hybrid Resonant Converters operating in discontinuous conduction mode using the phase shifting method will be described. Computer simulation to prove the feasibility of the phase shifting method in the design example will also be provided. Analysis of the characteristics of the output voltage produced by phase shifting control along with the steady state output analysis of the hybrid loaded resonant converters will also be discussed.
\end{abstract}

\section{INTRODUCTION}

Loaded resonant converters are typically used in highvoltage at medium power applications and have the advantages due to their soft-switching characteristics. Because the circuit can deliver the output power either intermittently or continuously, the loaded resonant converters may be operated in either continuous (CCM) or discontinuous conduction mode (DCM). The DCM operation allows for low switching losses, resulting from naturally commutating semiconductor devices under sinusoidal current waveforms. There are two basic topologies for the loaded resonant converters: series loaded resonant converter and parallel loaded resonant converter [2]. Each type possesses both advantages and drawback over the other. One topology that will combine the advantages of both types of the converter is the Hybrid Resonant Converter (HRC). HRC is a versatile topology because when used in DCM, it takes on the properties of both the Series Load Resonant Converter (SRC) and Parallel Load Resonant Converter (PRC). For instance, the HRC minimizes its switching losses since zero level current switching can be used while the converter operates at no-load [3]. The provision of current limiting under short-circuit condition while still acting as a voltage source is another benefit of using HRC [4].

In this paper the parallel operation of two HRCs both running in DCM is investigated. The output voltages from the converters are combined and varied to adjust the output power. Traditionally, the output power from loaded resonant converter is adjusted by varying the switching frequency. This method, while simpler, may be prone to audible noise when the frequencies used fall within audible range. This is not an acceptable condition for many applications such as the medical applications. The phase-shifting control scheme offers to overcome this potential problem with switching frequency control to adjust the output power. The phase shifting control entails the use of two $\mathrm{HRC}$ in parallel and to vary their combined output voltage by changing the 'phase' between the two converters. The output voltage may be varied from essentially zero, when the two converters are completely outof-phase, to twice the output voltage of one of the converters, when both converters are in phase. This outcome can be generated as long as the ac output voltage of each converter is combined before it is rectified and filtered

Operating two converters in parallel allows for a constant switching frequency as well as a constant duty-cycle. The constant switching frequency avoids the need to vary switching frequency and hence will prevent converter's operation in the audible range. The constant duty cycle gives the advantage of a simpler switching signal control circuitry.

In this paper, the design, analysis, and simulation of phase-shifting control in two HRC in parallel operating in DCM will be presented. The combined output voltage from both converters is varied using phase variation while maintaining the steady-state characteristics of the individual HRC. A comparison of frequency and phase variation, as well as any additional advantages and disadvantages of the design will also be discussed.

\section{ANALYSIS AND DESIGN}

Figure 1 shows the power stage of a dc-dc hybrid loaded resonant converter. The operation of the converter depends on how the resonant inductor current behaves: Continuous Conduction Mode (CCM) or Discontinuous Conduction Mode (DCM). For the best soft-switching characteristics, DCM is usually chosen over the CCM. The DCM occurs when there is a discontinuity in resonant inductor current, i.e. the current stays at zero for a finite amount of time. This discontinuity is the key to having an adjustable output. The longer time the resonant inductor stay at zero the less the output voltage will be. This type of control can be achieved by adjusting the switching frequency of the converter.

In this section, a design example involving two HRCs with half-bridge topology to produce $100 \mathrm{kV}$ and $10 \mathrm{~mA}$ at the output will be presented. Figure 2 shows the parallel connection of two HRCs. The $100 \mathrm{kV}$ was chosen since this would provide a 
good example for medical applications (such as x-ray generator). The $10 \mathrm{~mA}$ was selected to yield a $1 \mathrm{~kW}$ maximum output power of the converter which falls within the medium power category. The converters are designed to operate at 25 $\mathrm{kHz}$ in DCM with a three-phase $480 \mathrm{~V}_{\mathrm{rms}}$ input voltage. The 25 $\mathrm{kHz}$ was selected to be the switching frequency since it is above the audible frequency range for human. As shown in Figure 2, following the transformer, a multi-stage capacitordiode voltage multiplier is implemented to further boost the output voltage to the desired value. The output ripple will be minimized by using a filter to remove the $25 \mathrm{kHz}$ component.

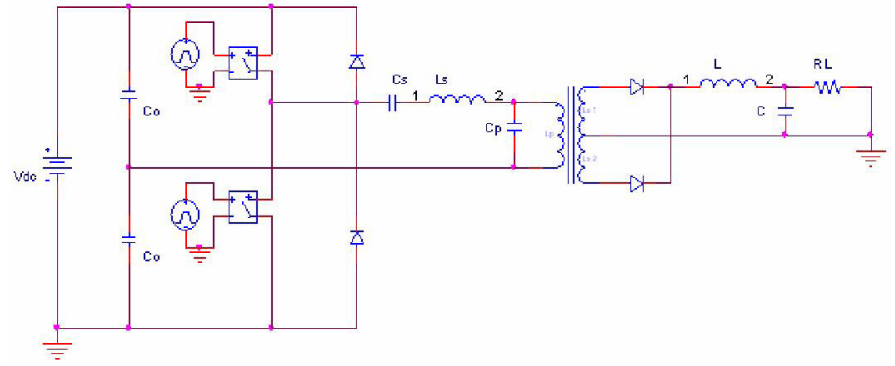

Figure 1. Hybrid Resonant Loaded Converters

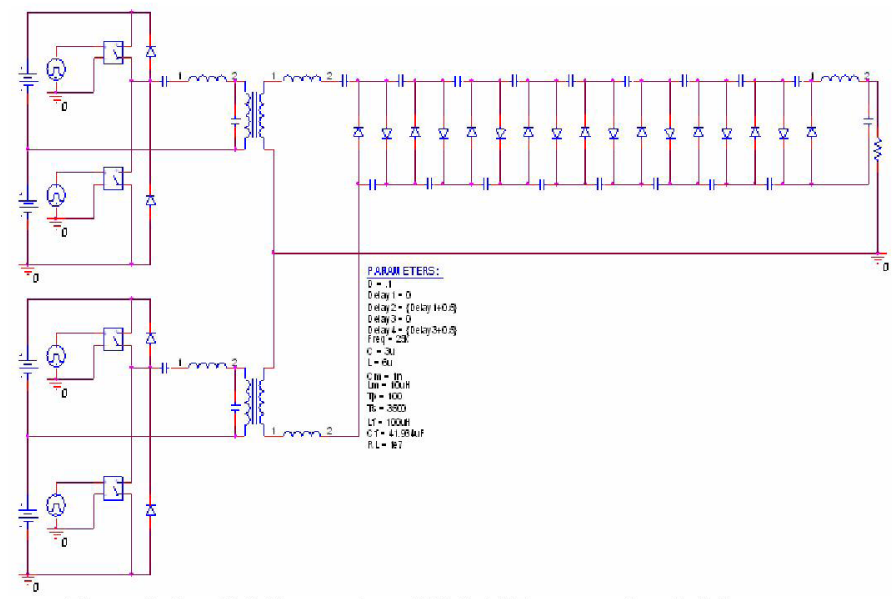

Figure 2. Parallel Connection of Hybrid Resonant Loaded Converters

\section{A. Input Stage}

The following equations are used to calculate the rectified dc voltage produced by a three-phase line-to-line $480 \mathrm{~V}_{\mathrm{rms}}$ input.

$$
\begin{aligned}
A_{d} & =\int_{-\pi / 6}^{\pi / 6} \sqrt{2} V_{L I} \cos w t \cdot d(w t) \\
& =\sqrt{2} V_{L I} \\
V_{d} & =\frac{A_{d}}{\pi / 3}=1.35 V_{L L}
\end{aligned}
$$

Equation (2) reveals that with a $480 \mathrm{~V}_{\mathrm{rms}}$ line to line voltage, the expected de input voltage to the converter is $1.35 * 480 \mathrm{~V}=$ $648 \mathrm{~V}$. Assuming the ripple of the dc input is very small, the dc input will therefore be represented by two de voltage sources in place of the dc-blocking capacitors and diode, generally found in the $\mathrm{HRC}$, with values of $321 \mathrm{~V}$ each. The following explains calculations of some of components in the circuit.

\section{B. Resonant Components}

The HRC has the ability to operate in three different modes: continuous conduction below the resonant frequency, continuous conduction above the resonant frequency, and discontinuous conduction. Due to more superior soft switching characteristics, the converter will be operated in DCM. The converter's switching frequency must then be less than half the resonant frequency $f_{0}$.

$$
f_{s}<f_{0} / 2
$$

The resonant frequency is calculated initially by finding the equivalent capacitance $\mathrm{C}$, of $\mathrm{C}_{\mathrm{s}}$ and $\mathrm{C}_{\mathrm{p}}$ connected in series [1] and seen in Figure 3.

$$
C=\frac{\left(C_{s} \cdot C_{p}\right)}{\left(C_{s}+C_{p}\right)}
$$

The equivalent inductance $\mathrm{L}$ would be calculated based on the series inductance and any parasitic inductances due to the transformer windings. However an ideal transformer model will be used in subsequent simulations, therefore $L_{s}$ will be assumed to equal $\mathrm{L}$.

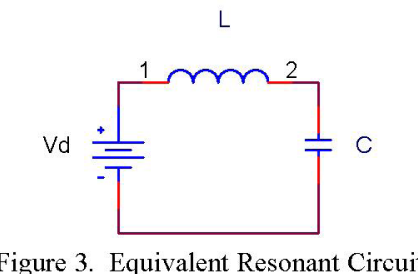

\section{Transformer Selection}

The challenge in selecting an appropriate transformer for simulation lies in selecting the proper turns-ratio to generate a large output voltage, while minimizing the leakage inductance which may help in increasing efficiency [1]. In order to reach an output voltage of roughly $100 \mathrm{kV}$, eight stages of doublers will be used (the multiplier circuit will be discussed more thoroughly in the next section) to supplement the converters and transformer, leading to a minimum output voltage of $391 \mathrm{~V}$ before the multiplier stage. The turn ratio will be based on knowing this fact, as well as the performance of the circuit.

\section{Multiplier and Filter Stage}

As shown in Figure 1, a multiplier circuit is being implemented to boost the output voltage. This derivation is used to take advantage of the phase-shifted voltage waveforms by summing the ac output waveforms from each converter before the multiplier circuit, thus utilizing phase shifting to control the output voltage level. To keep the turns ratio close to a practical value, it was found that 17 stages of the Capacitor- 
Diode are needed to get the required output voltage as shown in Figure 2.

To reduce a voltage ripple at the multiplier output, an LC low-pass filter was added as a final stage to the multiplier circuit. The following is a design procedure used to integrate a filter capable of eliminating the converter's switching frequency of $25 \mathrm{kHz}$. The transfer function values of the inductance and capacitance can be found in terms of frequency. With the attenuation set to 40 decibels, the frequency to 25 $\mathrm{kHz}$, either the inductance value or the capacitance value can be used as to the remaining variable. The following equations demonstrate the process mathematically.

$$
\begin{aligned}
& \frac{V_{\text {out }}}{V_{\text {in }}}=\left[\frac{1 / j \omega C}{j \omega L+1 / j \omega C}\right] \cdot\left(\frac{j \omega C}{j \omega C}\right) \\
& \left|V_{\text {out }} / V_{\text {in }}\right|=\frac{1}{\omega^{2} L C-1} \\
& \text { decibels }=20 \log \left|V_{\text {out }} / V_{\text {in }}\right|
\end{aligned}
$$

Using equation (7), we can then determine the filter capacitor value given the switching frequency and filter inductor value. As an example, with a switching frequency $\left(\boldsymbol{f}_{\mathrm{s}}\right)$ of $25 \mathrm{kHz}$ and filter inductance $\left(\mathrm{L}_{\mathrm{f}}\right)$ of $100 \mu \mathrm{H}$, the filter capacitance $\left(\mathrm{C}_{\mathrm{f}}\right)$ is equal to $40.934 \mu \mathrm{F}$.

\section{SIMULATION RESULTS}

Simulation results will be used to verify the following: the design is operating in DCM, the output voltage can be varied by phase-shifting, and that the inherent characteristics of the HRC are maintained. Additionally, analysis of any advantages and disadvantages of the design will be brought to attention. To accomplish these objectives, a computer simulation of the circuit shown in Figure 1 was conducted using OrCAD schematics.

Operating the HRC in DCM allows the switch to be operated so the voltage across and or the current through it is zero at the switching instant, eliminating switching losses. The first obstacle in simulating the converter then, is to ensure its operation in DCM at the desired switching frequency of 25 $\mathrm{kHz}$.

Parallel operation can be used to change the output voltage by changing the 'phase' between two converters. This is done by introducing a delay angle in the switching control signal to one of the converter such that the resonant inductor current of one converter as shown in Figure 1 will be lagging with respect to the resonant inductor current of the other converter. As an example, Figure 4 shows the resonant inductor current at zero degree while Figure 5 shows the same current at 180 degrees. Theoretically, adding the two currents will yield a zero output current and hence zero output voltage or power. Figures 6 and 7 show that the output voltage when the phase-shifts are zero and 144 degrees respectively. These phase shifts in turn may produce an output voltage that can be varied from $22 \mathrm{kV}$ to $100 \mathrm{kV}$.

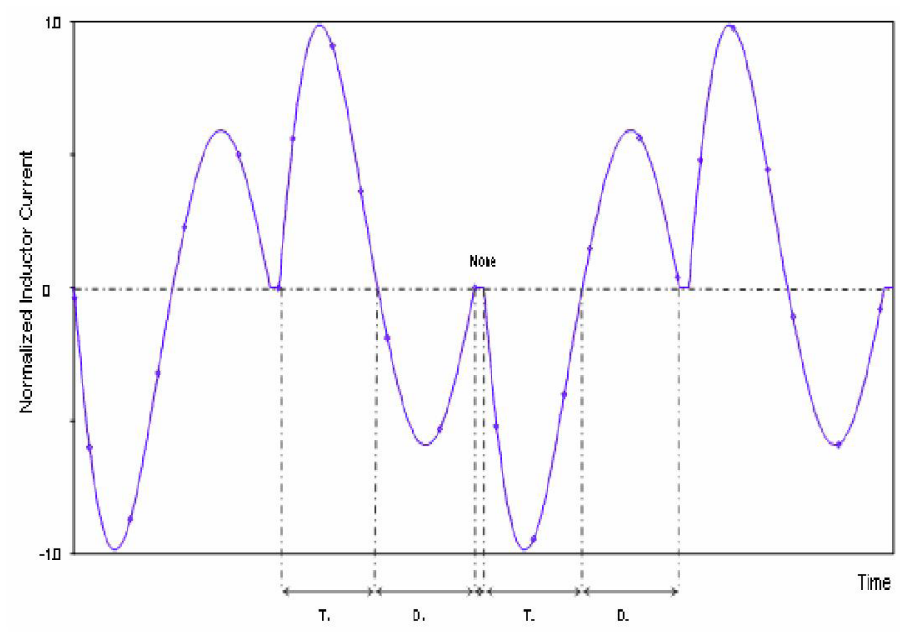

Figure 4. Resonant Inductor Current showing DCM operation at 0 degree

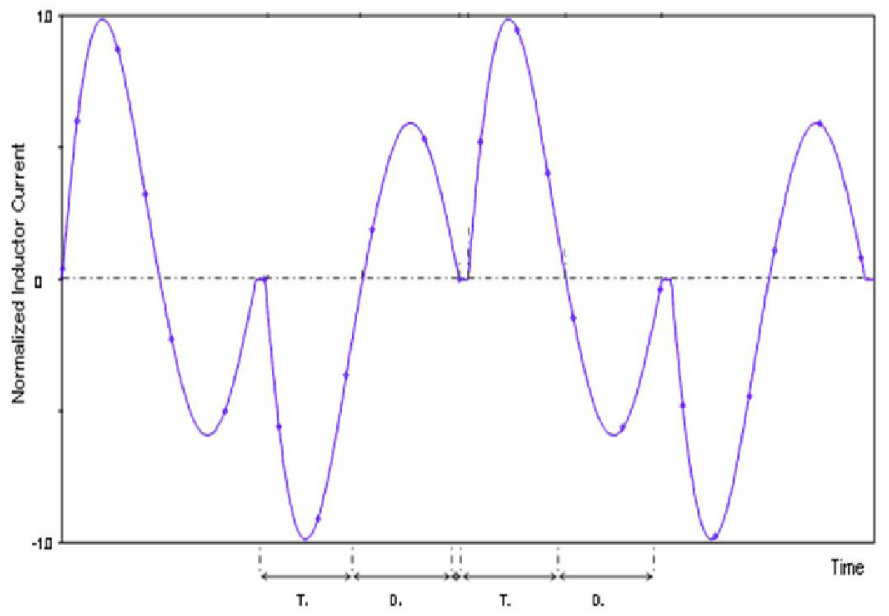

Figure 5. Resonant Inductor Current showing DCM operation at $180^{\circ}$

Frequency variation is traditionally used as the method to control the output voltage level. Assuming the converters were to use frequency variation, it would operate at frequencies lower than $1 / 2 f_{\mathrm{s}}$ to vary the output voltage from $22 \mathrm{kV}$ to $110 \mathrm{kV}$ and still remain in DCM. Figure 8 plots the output power vs. both switching frequency control and phase-shift control. In order to maintain the output power within the range produced by phase-variation, the frequency must be varied between 10 and $25 \mathrm{kHz}$. This poses a major problem since it overlaps well with the audible frequency range (10 to $20 \mathrm{kHz})$. The switching would occur far from 3.5 to $4 \mathrm{kHz}$ range the human ear has peak sensitivity to; however, switching would occur near another enhanced sensitivity region at about $13.5 \mathrm{kHz}$ [5].

With the converter operating at a switching frequency of $25 \mathrm{kHz}$ the design has both the excellent voltage regulation known to PRC as shown in Figure 9 and the current limiting 
feature known to SRC as shown in Figure 10. Figure 9 shows when the design is operated in DCM, it acts as a voltage source as evidenced from the merging plots. This means that although the load resistance and therefore load current was changed, the circuit will still maintain its rated output voltage, i.e. ideal load regulation. As in any other power supply, a very good load regulation is desired to maintain a stable output voltage at various load power condition. Figure 9 further illustrates that when the converter began to operate in continuous conduction mode, the load regulation worsened as exhibited by the circuit's performance beyond $\mathrm{w}_{\mathrm{s}} / \mathrm{w}_{\mathrm{o}}=0.5$ and as evidenced by the splitting plots.

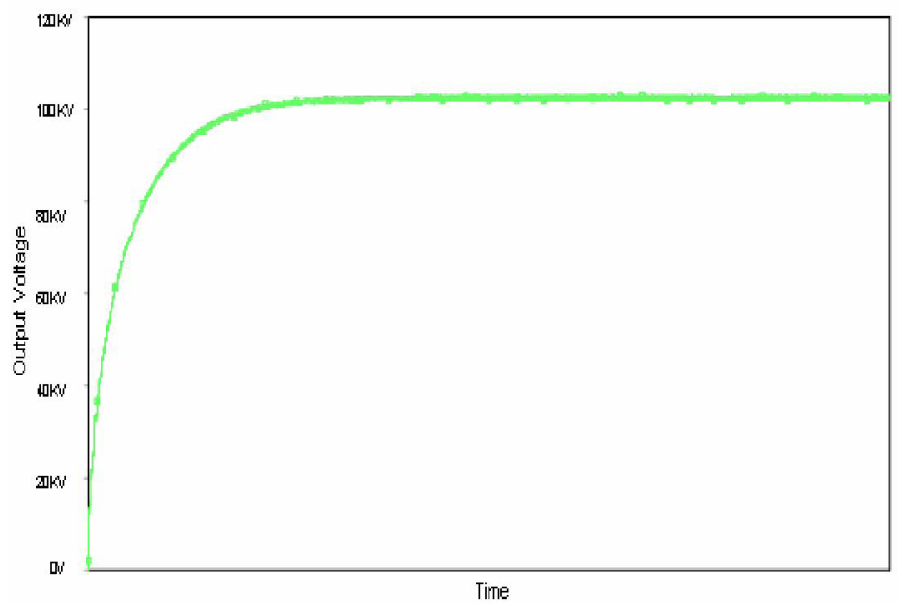

Figure 6. Output Voltage with phase-shift of $0^{\circ}$

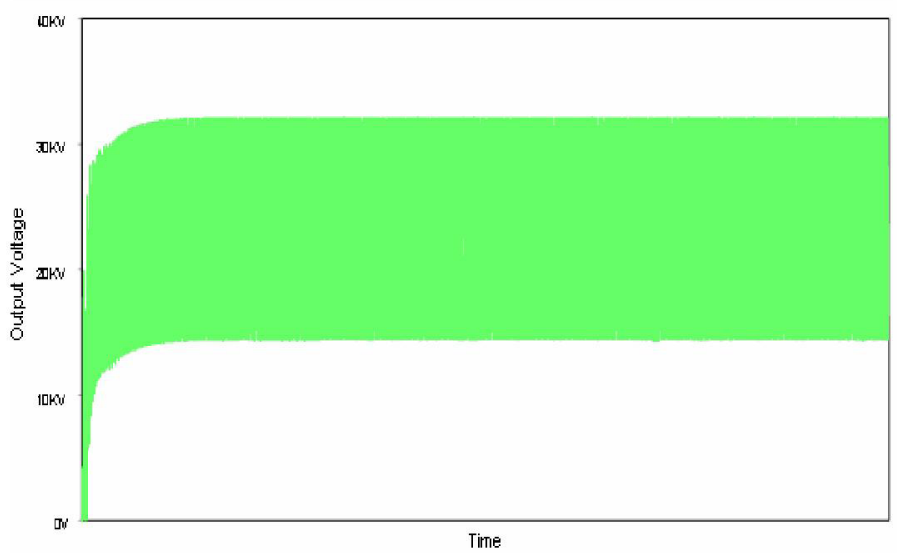

Figure 7. Output Voltage with phase-shift of $144^{\circ}$

Figure 10 shows the current limiting characteristics of the HRC. To obtain this, a dc voltage source was used to model the load such that the output voltage can be varied to get the output current data. Unlike when the design operates in $\mathrm{CCM}$, in DCM the design limits the current flow through the load independent of output voltage. This current limiting characteristic means that the converter has an inherent short circuit protection. This would simplify output or load protection circuitry.

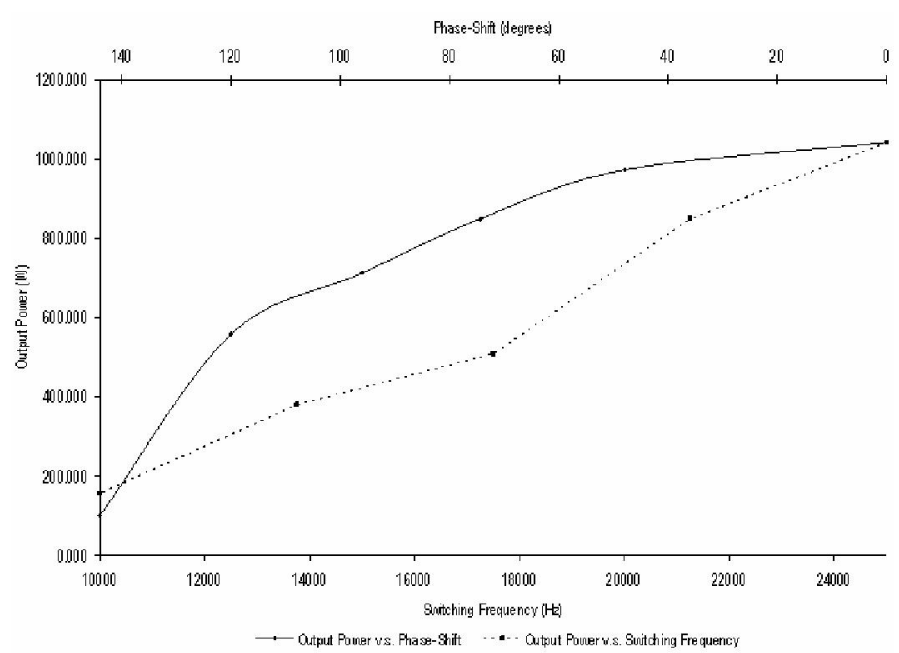

Figure 8. Output Voltage vs. Phase-Shift \& Switching Frequency

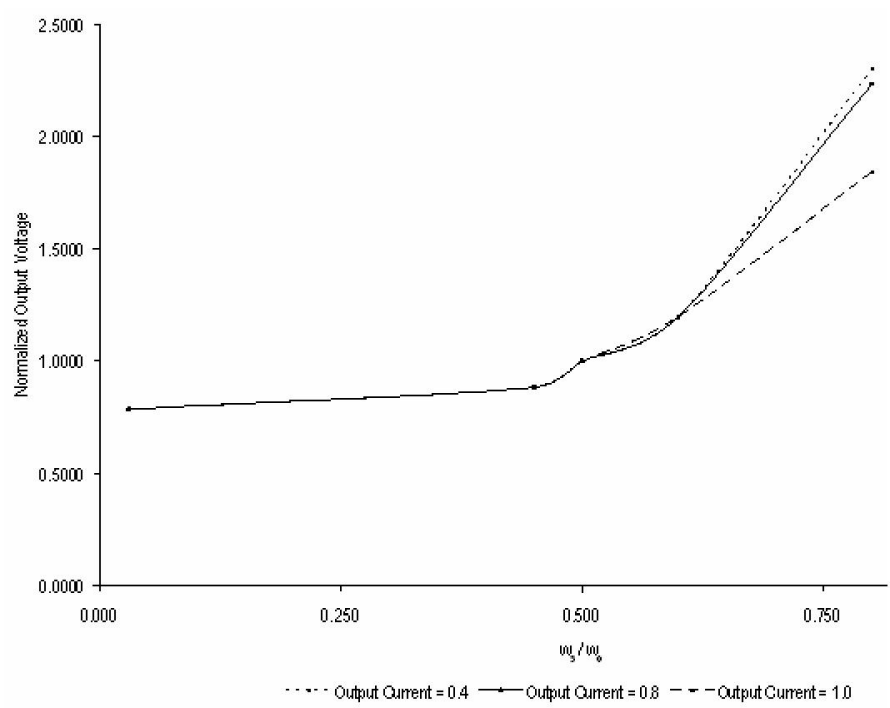

Figure 9. Voltage Source Characteristic of the HRC

Although to this point, phase variation appears to be advantageous, it has its problems, namely its effect on the output ripple. When comparing Figure 6 to 7 it is evident that the output ripple is significantly larger when the phase between the two converters is increased. Figure 11 shows that for an increase in phase, there is a near linear increase in output ripple. Furthermore, total harmonic distortion of the output voltage was also measured and it is larger as the phase shift is increased as shown in Figure 12. Fourier analysis of the output current shows that as the phase is increased, the dc component becomes smaller in magnitude. Consequently, components at higher frequencies are larger, relative to the dc component, leading to a larger ripple. Another disadvantage to this methodology is the need for two converters and consequently twice the components. However, each converter could be designed for only half of the voltage requirement necessary for the overall system. 


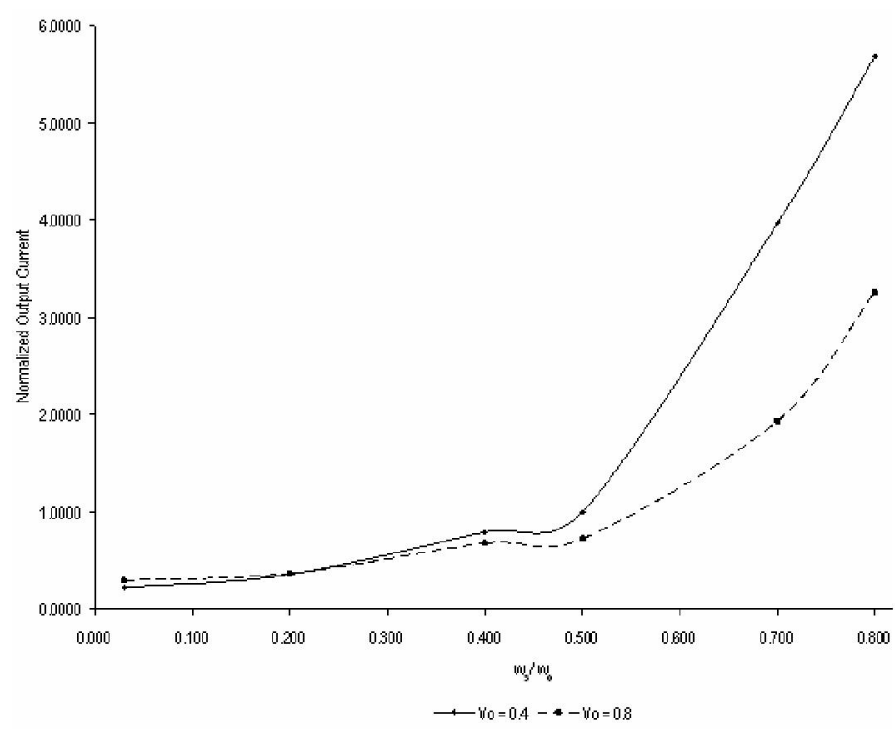

Figure 10. Current Limiting Characteristic of the HRC

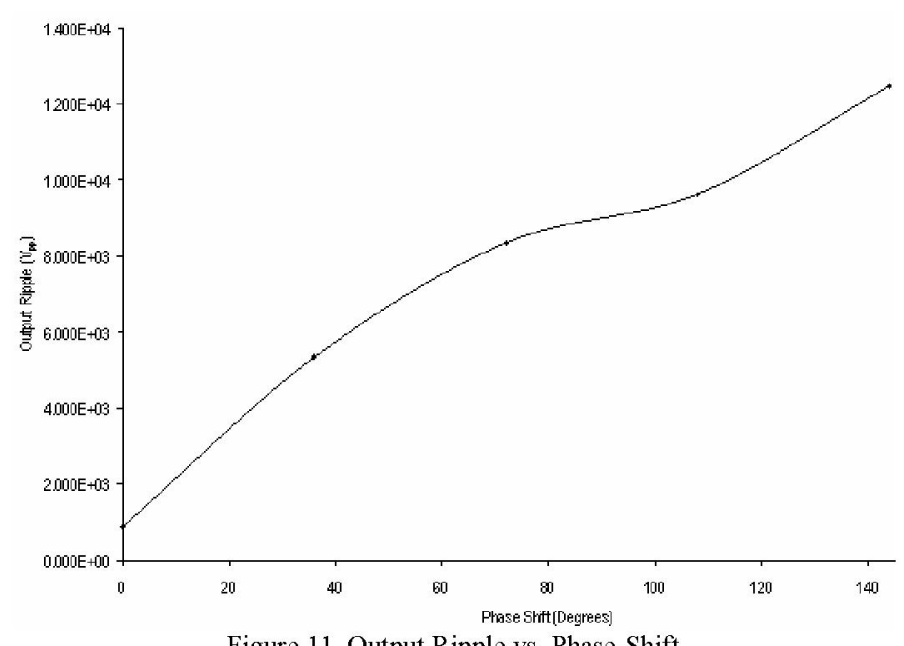

Figure 11. Output Ripple vs. Phase-Shift

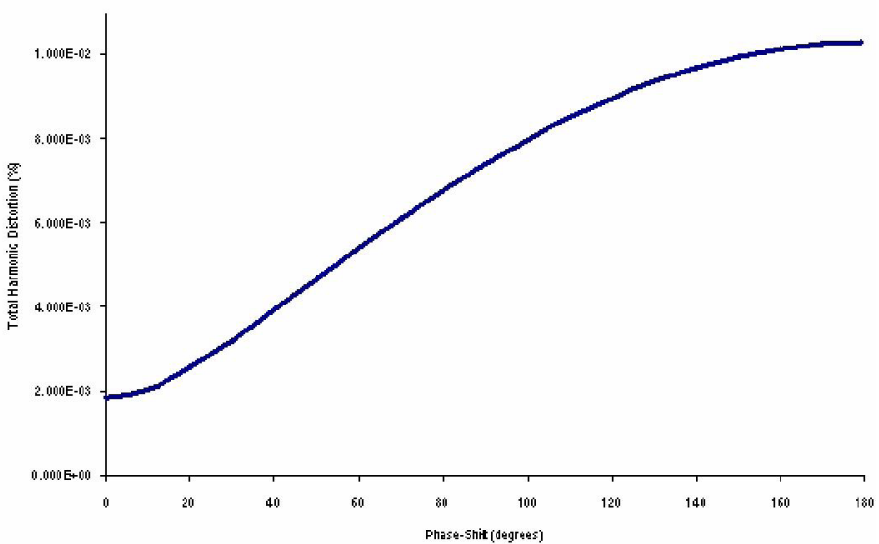

Figure 12. THD of output voltage vs. Phase-Shift

\section{CONCLUSIONS}

In this paper, the phase shifting control to adjust the output voltage of two Hybrid Resonant loaded converters connected in parallel and operating in discontinuous conduction mode has been demonstrated. The design and analysis were first presented and the phase-shifting control was then verified using computer simulation. The result shows that the phase shifting control is able to provide a smooth output voltage adjustment while the converter's switching frequency remains the same. Furthermore, the result also shows that the hybrid converter retains the benefits coming from both the series and the parallel loaded resonant circuits.

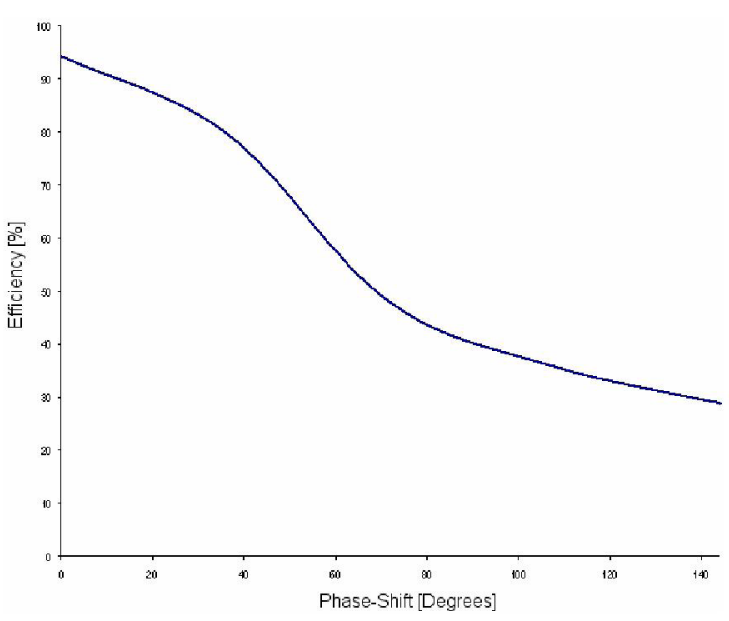

Figure 13. Converter's Efficiency vs. Phase-Shift

There are however disadvantages associated with the phase shifting method. The combined output voltage of the parallel hybrid converters exhibited an increase in output ripple when the phase control was increased between them. This disadvantage was shown to be caused by the reduction in the magnitude of the $\mathrm{dc}$ component as phase was increased. Consequently, the overall efficiency of the converter will also worsen as phase-shift is increased as shown in Figure 13 .. Nevertheless, the parallel hybrid converters with phase-shifting control provide an effective alternative to frequency-variation and other resonant topologies in applications where audible noise is a concern. Further work is currently undergoing to build a $1-\mathrm{kV}$ hardware prototype of the circuit to further prove the phase-shifting control. Results of this hardware test will be reported in future ISIE conference.

\section{REFERENCES}

[1]. Chen, W., "Series-Parallel Resonant Forward Inverter as a Cold Cathode Fluorescent Lamp (CCFL) Driver," Applied Power Electronics Conference and Exposition, 133-137, 2002.

[2]. G.D. Demetriades, "Power Resonant Converters", Proceedings of the Power Electronics and Industrial Electronics Conference, Athens, Greece, 2000.

[3]. Johnson, S.D., Witulski, A.F. \& Erickson, R.W., "A Comparison of Resonant Topologies in High Voltage Applications." IEEE Trans. on Aerospace and Electronic Systems, Vol. AES-24, No. 3, 263-274., 1988.

[4]. Mohan, N., Undeland, T., \& Robbins, W., Power Electronics: Converters, Application and Design. John Wiley and Sons Inc., 1989.

[5]. Nave, C.R., Hyper Physics [Online Web site]. Available WWW: http:/hyperphysics.phy-astr.gsu.edu 